\title{
An Advanced Apparatus for Integrating Nanophotonics and Cold Atoms
}

\author{
Zhongzhong Qin, Jean-Baptiste Béguin, Alexander Burgers, Xingsheng Luan, \\ Su-Peng Yu, H. Jeff Kimble ${ }^{\dagger}$ \\ Norman Bridge Laboratory of Physics, California Institute of Technology, Pasadena, California 91125, USA \\ $\dagger$ hjkimble@caltech.edu
}

\begin{abstract}
Integrating nanophotonics with cold atoms permits the exploration of novel paradigms in quantum optics and many-body physics. We realize an advanced apparatus which enables the delivery of single-atom tweezer arrays in the vicinity of photonic crystal waveguides. (c) 2020 The Author(s)
\end{abstract}

Integrating nanophotonics and cold atoms has drawn increasing interest in recent years due to its diverse applications in quantum information science and the exploration of quantum many-body physics [1]. For example, dispersion-engineered photonic crystal waveguides (PCWs) permit not only stable trapping and probing of ultracold neutral atoms via interactions with guided mode light, but also the possibility to study the physics of strong, photon-mediated interactions between atoms, as well as atom-mediated interactions between photons. Even though diverse theoretical opportunities involving atoms and photons in 1-D and 2-D nanophotonic lattices have been proposed, it still remains challenging to experimentally integrate nanophotonics devices with the realm of unltacold atoms [2]. We have developed an advanced apparatus that overcomes several significant barriers to current experimental progress for the integration of cold atoms and nanophotonics [3]. Our goal is to achieve strong quantum interactions of light and matter by way of single atoms and photons in 1-D and 2-D nanophotonic structures to enable diverse exciting physics exploration. Our new apparatus has the capability to deterministically assemble atomic arrays near the surfaces of 1-D and 2-D PCWs as illustrated schematically in Fig. 1.

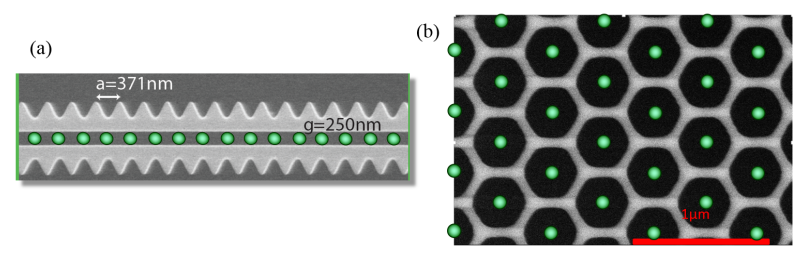

Fig. 1. Schematic for building nanoscopic atomic arrays with one atom per unit cell in (a) 1-D alligator PCW and (b) 2-D honeycomb lattice PCW, respectively.

Our apparatus is shown in Fig. 2 (a), which utilizes a silicate bonding technique [4] to secure a Si chip to an $\mathrm{SiO}_{2}$ glass cell with an inner wall spacing of $1 \mathrm{~cm}$ for large optical access and ultra-high vacuum operation. The $\mathrm{Si}$ chip contains 16 PCWs, and is silicate bonded on a $\mathrm{SiO}_{2}$ table as shown in Fig. 2(b), which is then silicate bonded on the inner wall of the glass cell. With the unprecedented optical access and small size of the glass cell, two coupling objectives to PCWs and two tweezer objectives are utilized around the glass cell. All four objectives have numerical aperture (NA) of 0.4. The tweezer beam has a waist of $1.26 \mu \mathrm{m}$, and sub-micron waists have also been achieved with higher NA objectives.

Cold atoms are delivered to the glass cell along its vertical axis by a blue-detuned "donut" guiding beam, and then recaptured and cooled by Polarization Gradient Cooling (PGC) to a temperature $\simeq 20 \mu \mathrm{K}$. The PGC cloud is around $3 \mathrm{~mm}$ away from the surface of the Si chip to minimize the scattering from the chip. Next, individual Cesium atoms from the PGC cloud are loaded into an optical tweezer array operated at $936 \mathrm{~nm}$ for Cs deep in a regime of "collisional blockade", as shown in Fig. 3(a). Atomic fluorescence imaging of atoms loaded into freespace tweezer array for 100 experimental shots is shown in Fig. 3(b). The reflection of sixteen tweezer spots off the PCW is shown in Fig. 3(c), which is imaged by the tweezer objective. Long lifetime up to 25 seconds for trapped atoms in the tweezer array allows us to deterministically couple single atom array in the vicinity of the PCWs by moving tweezer objective on a precision linear translation stage. Furthermore, the power handling capabilities of the free-space PCWs with novel design have increased to $\sim 10 \mathrm{~mW}$ from $\sim 0.5 \mathrm{~mW}$ for our previous butt-coupled 
(a)

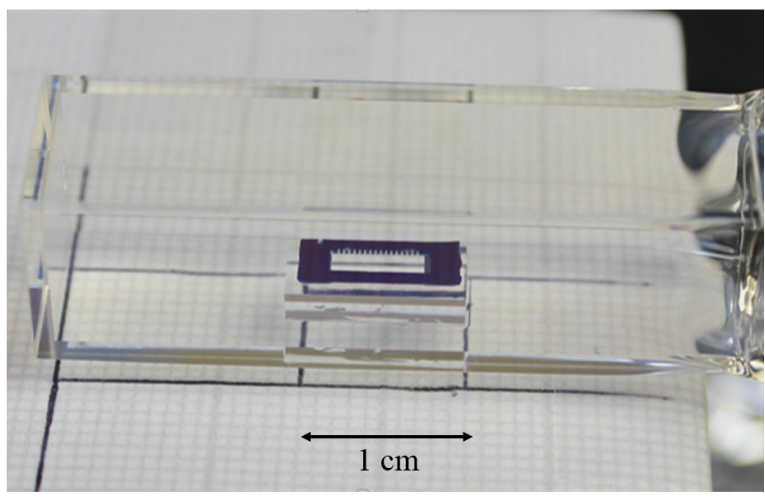

(b)

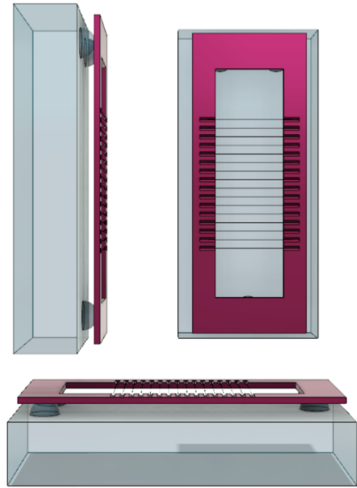

Fig. 2. (a) Our apparatus for deterministically assembling atom array near PCWs. (b) 3-D model drawings of the $\mathrm{SiO}_{2}$ 'table' assembly with Silicon chip, which is in turn mounted inside the $\mathrm{SiO}_{2}$ glass cell. The chip contains $16 \mathrm{Si}_{3} \mathrm{~N}_{4}$ PCWs which are suspended across a $2 \mathrm{~mm} \times 7 \mathrm{~mm}$ window.

devices. This will enable longer lifetimes for guided-mode traps by way of higher intensities required for larger atomic detunings, including for magic-wavelength traps.

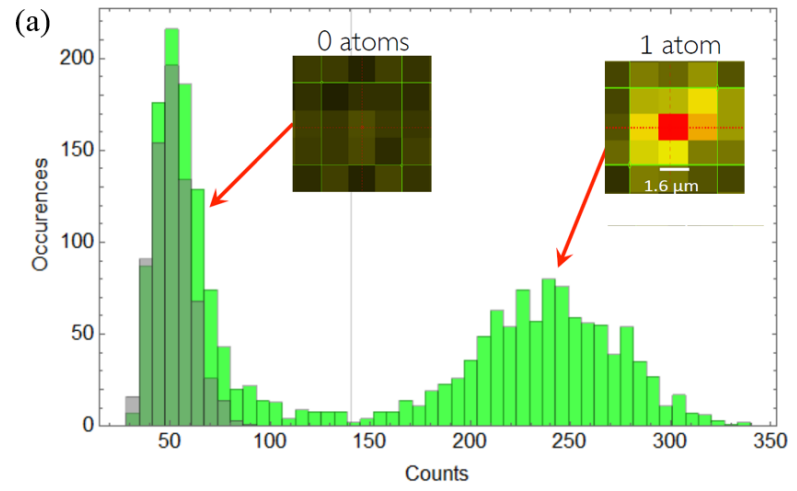

(b)

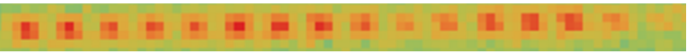

(c)

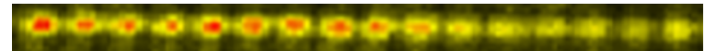

Fig. 3. (a) Fluorescence histogram of single atom in optical tweezer and no atom in tweezer. Single atom loading probability is 0.488 . Insets show the images for 0 atoms and 1 atom, respectively. (b) 100-shot averaged free-space atomic fluorescence imaging from loading of the sixteen tweezer sites at a distance of $3 \mathrm{~mm}$ away from the chip. (c) Scattering of the sixeen tweezer spots on the nanophotonic waveguide.

\section{References}

1. D. E. Chang, J. S. Douglas, A. González-Tudela, C. L. Hung, and H. J. Kimble, "Colloquium: Quantum matter built from nanoscopic lattices of atoms and photons," Rev. Mod. Phys. 90, 031002 (2018).

2. T. Tiecke, J. D. Thompson, N. Pulmones de Leon, L. R. Liu, V. Vuletić, and M. D. Lukin, "Nanophotonic quantum phase switch with a single atom," Nature 508, 241 (2014).

3. J.-B Béguin, A. P. Burgers, X. Luan, Z. Qin, S. P. Yu, and H. J. Kimble, “ Advanced apparatus for the integration of nanophotonics and cold atoms," Optica 7, 1 (2020).

4. A.-M. A. van Veggel and C. J. Killow, "Hydroxide catalysis bonding for astronomical instruments," Adv. Opt. Technol. 3, 293 (2014). 